\title{
In the back of your mind: Cortical mapping of tactile and proprioceptive paraspinal afferent inputs
}

David M. Cole ${ }^{1-3}$, Philipp Stämpfli ${ }^{3,4}$, Robert Gandia ${ }^{5}$, Louis Schibli ${ }^{5}$, Sandro Gantner ${ }^{5}$, Philipp

$$
\text { Schuetz }^{5} \text {, Michael L. Meier }{ }^{1,2 *}
$$

${ }^{1}$ Department of Chiropractic Medicine, Integrative Spinal Research, Balgrist University Hospital, University of Zurich, Zurich, Switzerland

${ }^{2}$ University of Zurich, Zurich, Switzerland

${ }^{3}$ Department of Psychiatry, Psychotherapy and Psychosomatics, Hospital of Psychiatry, University of Zurich, Zurich, Switzerland

${ }^{4}$ MR-Center of the Psychiatric Hospital, University of Zurich, Zurich, Switzerland

${ }^{5}$ Competence Center Thermal Energy Storage, Lucerne University of Applied Sciences and Arts, Lucerne, Switzerland

Corresponding author $(*)$ :

Michael L. Meier, Balgrist University Hospital, Department of Chiropractic Medicine, Forchstrasse 340, 8008 Zurich, Switzerland; E-Mail: michael.meier@balgrist.ch; Phone: +41 44 5107380

Number of words: 5,902; Number of Tables: 3; Number of Figures: 5

Keywords: fMRI, somatosensory cortex, proprioception, back, motor control 


\section{Abstract}

Persistent pain alters brain-body representations, highlighting their potential pathological significance. In chronic low back pain (LBP), sparse evidence points towards a shift of the cortical representation of sensory afferents of the back. However, systematic investigations of the cortical representation of tactile and proprioceptive paraspinal afferents along the thoracolumbar axis are lacking. Detailed cortical maps of paraspinal afferent input might be crucial to further explore potential relationships between brain changes and the development and maintenance of chronic LBP. We therefore validated a novel and functional magnetic resonance imaging(fMRI-)compatible method of mapping cortical representations of tactile and proprioceptive afferents of the back, using pneumatic vibrotactile stimulation ("pneuVID") at varying frequencies and paraspinal locations, in conjunction with high-resolution fMRI. We hypothesised that: (i) high $(80 \mathrm{~Hz})$ frequency stimulation would lead to increased postural sway compared to low $(20 \mathrm{~Hz})$ stimulation, due to differential evoked mechanoreceptor contributions to postural control (proprioceptive vs tactile); and (ii) that high $(80 \mathrm{~Hz})$ versus low $(20 \mathrm{~Hz})$ frequency stimulation would be associated with neuronal activity in distinct primary somatosensory (S1) and motor (M1) cortical targets of tactile and proprioceptive afferents $(\mathrm{N}=15$, healthy volunteers). Additionally, we expected neural representations to vary spatially along the thoracolumbar axis. We found significant differences between neural representations of low and high frequency stimulation and between representations of thoracic and lumbar paraspinal locations, in several bilateral sensorimotor cortical regions. Proprioceptive $(80 \mathrm{~Hz})$ stimulation preferentially activated sub-regions S1 3a and M1 4p, while tactile (20 Hz) stimulation was more encoded in S1 3b and M1 4a. Moreover, in S1, lower back proprioceptive stimulation activated dorsal-posterior representations, compared to ventral-anterior representations activated by upper 
back stimulation. As per our hypotheses, we found distinct sensorimotor cortical tactile and proprioceptive representations, with the latter displaying clear topographic differences between the upper and lower back. This thus represents the first behavioural and neurobiological validation of the novel pneuVID method for stimulating muscle spindles and mapping cortical representations of paraspinal afferents. Future investigations of detailed cortical maps will be of major importance in elucidating the role of cortical reorganization in the pathophysiology of chronic LBP. 


\section{Introduction}

One of the most pervasive chronic health conditions is low back pain (LBP), which carries a lifetime prevalence of around 75-84\% (Thiese et al., 2014). Globally, chronic LBP is the leading health condition in terms of the total number of years lived with a disability (Vos et al., 2017). In most cases, no underlying pathology can be identified for instances of LBP (Maher et al., 2017). Current evidence suggests that a strict therapeutic and research focus on the spine and its mechanics is only moderately successful in alleviating the global disease burden, suggesting that supraspinal mechanisms such as changes in sensorimotor integration contribute to the development and maintenance of chronic LBP (Wand et al., 2011; Moseley and Flor, 2012; Massé-Alarie and Schneider, 2016; Jenkins et al., 2021; Langevin, 2021).

The central processing of proprioceptive input is an essential component of sensorimotor integration and, therefore, proprioceptive impairment can contribute substantially to motor control dysfunction in LBP (Van Dieën et al., 2017, 2019; Meier et al., 2018). At the behavioural level, chronic LBP patients demonstrate impaired lumbar proprioception compared to healthy controls (Brumagne et al., 2008; Lee et al., 2010; Tong et al., 2017), indicating altered paraspinal muscle spindle signalling and/or central processing of this sensory input. Proprioceptor function is principally served by muscle spindles residing in the muscle belly, parallel to the extrafusal fibres, while auxiliary functions are provided by sensory receptors located in joints, ligaments, tendons, fascia and the skin (Proske and Gandevia, 2012). Muscle spindle function appears highly important for both proprioception and trunk motor control and can be manipulated by applying vibratory stimulation to muscles and/or tendons at frequencies of approximately $80 \mathrm{~Hz}$ (and amplitudes of 0.5-1.0 mm; Brumagne et al., 2000; Claeys et al., 2010). Such stimulation 
protocols have been shown to provoke an artificial lengthening of the muscle spindles which can produce both limb movement and positional illusions, thus impacting proprioceptive function (Goodwin et al., 1972; Burke et al., 1976; Gilman, 2002; Brumagne et al., 2008; Proske and Gandevia, 2012). Conversely, a lower-frequency vibratory stimulus of around $20 \mathrm{~Hz}$ preferentially activates mechanoreceptors in superficial skin layers (e.g., Meissner's corpuscles) involved in tactile somatosensory functioning, rather than muscle spindles (Weerakkody et al., 2007). Therefore, vibrotactile stimulation at varying frequencies can be used to disentangle the central processing of different mechanoreceptor inputs (Avanzino et al., 2014). Although the proprioceptive input axis has rarely been studied in the context of LBP, the primary somatosensory cortex (S1), which is the major cortical target of proprioceptive information (Riemann and Lephart, 2002; Kim et al., 2015), is likely to play a key part given its crucial role in sensorimotor integration and in the persistence of pain (Bingel et al., 2004; Lee et al., 2013; Massé-Alarie and Schneider, 2016; Makin and Bensmaia, 2017; Meier et al., 2018; Jenkins et al., 2021).

Chronic pain can alter cortical organization, a phenomenon that may carry pathophysiological relevance in this regard (Flor, 2003; Apkarian et al., 2005; Moseley and Flor, 2012). Indeed, in chronic LBP patients, differences of paraspinal muscle representations in the primary motor cortex (M1) have been observed in comparison to healthy controls, characterized by an increased overlap of the longissimus and deep multifidus muscles, indicating less fine-grained ("smudged") cortical representations of paraspinal muscles (Tsao et al., 2011b). Notably, this smudging might contribute to dysfunctions of motor control, which have been associated with the persistence of LBP (Van Dieën et al., 2017, 2019; Meier et al., 2018; Elgueta-Cancino et al., 2021). However, less is known about the extent to which such alterations in M1 motor maps are 
caused by changes from the 'input side', i.e., the processing of paraspinal sensory inputs, which must be similarly organized to allow for appropriate spinal movement (Riemann and Lephart, 2002; Hodges et al., 2013). More than two decades ago, researchers detected a shifted sensory representation of tactile input from the back in chronic LBP patients in the primary somatosensory cortex (S1) using magnetencephalography (MEG; Flor et al., 1997). However, this study has never been replicated and detailed cortical topographical maps of paraspinal sensory input along the thoracolumbar axis are lacking. Such maps are potentially crucial to enable further exploration of potential relationships between brain changes and the development and maintenance of chronic LBP (Schmid et al., 2021). In this regard, altered proprioceptive inputs from paraspinal muscles might be more important pathophysiologically - relative, for example, to tactile sensory inputs (Beaudette et al., 2016) - for the chronification of LBP. Nevertheless, the cortical targets and topographical organization of proprioceptive and tactile afferent inputs along the thoracolumbar axis have not yet been systematically investigated and differentiated.

We here sought to validate a technique combining vibrotactile stimulation of multiple paraspinal locations along the thoracolumbar axis, using a novel pneumatic vibration device ("pneuVID"; (Schibli et al., 2021) in conjunction with high-resolution brain functional magnetic resonance imaging (fMRI), in order to investigate such key questions of the cortical topography of paraspinal tactile and proprioceptive inputs, which hitherto remain unanswered. We hypothesised that high-frequency pneuVID stimulation ( $80 \mathrm{~Hz}$ vibration) triggering muscle spindle activity (i.e., proprioceptive input) would lead to increased postural sway and activate different neural representations of paraspinal afferent input compared to lower-frequency stimulation evoking a purely tactile sensory experience (20 Hz vibration). In addition, we aimed to differentiate a more 
bioRxiv preprint doi: https://doi.org/10.1101/2021.12.19.473341; this version posted December 21, 2021. The copyright holder for this preprint (which was not certified by peer review) is the author/funder, who has granted bioRxiv a license to display the preprint in perpetuity. It is made available under aCC-BY-NC-ND 4.0 International license.

finely-grained topographic map in S1 of back somatosensory representations along the thoracolumbar axis than has previously been attainable. 


\section{Methods}

\section{1. pneuVID}

\subsection{1. pneuVID system}

PneuVID was used to apply vibrotactile stimulation to different thoracolumbar segments. Details about the design, the different system components, and integration of pneuVID in the MR environment are described elsewhere (Schibli et al., 2021). In short, pneuVID can apply reliable vibrotactile stimulations with different frequencies and amplitudes in supine subjects during MR scanning (Fig. 1).

\subsection{2. pneuVID validation using postural sway data}

To assess the ability of pneuVID to stimulate muscle spindles and therefore modulate proprioceptive information, we used vibrotactile stimulation in combination with a force plate to track postural sway. Vibration applied at frequencies of $\sim 80 \mathrm{~Hz}$ (and amplitudes between 0.5-1.0 $\mathrm{mm}$ ) to tendons and/or muscles has been shown to artificially signal muscle lengthening (mediated through primary (Ia) muscle spindle afferents), which can provoke corrective movements (Roll and Vedel, 1982; Vedel and Roll, 1982; Brumagne et al., 2000; Seizova-Cajic et al., 2007). Furthermore, it has been proposed that during regular standing on a force plate with the feet next to each other, balance in the anterior-posterior direction is regulated predominantly by muscle contractions around the ankle (Winter, 1995). In support of this, using electric vibration units, it has been shown that $\sim 80 \mathrm{~Hz}$ vibration applied at the triceps surae muscles (mTS) during regular standing often leads to corrective movements, expressed by changes of the 
center of pressure (COP) in the anterior-posterior direction (path length over time; Brumagne et al., 2000; Claeys et al., 2015; Kiers et al., 2015). Therefore, using pneuVID, we hypothesized that $80 \mathrm{~Hz}$ vibration applied on the mTS would lead to increased postural sway compared to control frequencies such as $20 \mathrm{~Hz}$ (which should primarily activate mechanoreceptors in superficial skin layers, e.g., Meissner's corpuscles) and $0 \mathrm{~Hz}$ (Gandevia et al., 1992; Weerakkody et al., 2007; Proske and Gandevia, 2012). In addition, we explored whether different air pressures $(0.8 \mathrm{bar} / 1.5 \mathrm{bar})$ and amplitudes $(0.5 \mathrm{~mm} / 0.7 \mathrm{~mm}$, measured with a laser distance sensor, Welotec OWLF 4007 FA S1, Welotec GmbH, Laer, Germany) influenced a potential effect of $80 \mathrm{~Hz}$ vibration on postural sway.

\subsubsection{Postural sway validation: participant recruitment and study design}

For the postural sway assessments, we recruited a separate cohort of 15 healthy participants (one female, mean age $=28.5, \mathrm{SD} \pm 9.1)$. Exclusion criteria were excessive consumption of alcohol or consumption of other drugs or analgesics within the last $24 \mathrm{~h}$, prior foot or ankle surgery, any neuromuscular diseases that might affect gait and posture, injuries of the motor system with permanent deformities and body mass index $(\mathrm{BMI})>30 \mathrm{~kg} / \mathrm{m}^{2}$. The study was approved by the Ethics Committee Zurich (Switzerland, ID 2018-01001) and all patients provided written informed consent before participation. The study was conducted in accordance with the Declaration of Helsinki.

Prior to postural sway assessments, two pneuVID units were attached bilaterally on the mTS with adhesive tape. Subsequently, participants were instructed to stand on a force plate (Kistler 9260AA, Kistler Group, Winterthur, Switzerland) with their arms hanging loosely on the side. Furthermore, they were asked to wear headphones, and their vision was occluded using non- 
transparent goggles. Participants then underwent a stimulation protocol including the three different frequency conditions $(0 \mathrm{~Hz} / 20 \mathrm{~Hz} / 80 \mathrm{~Hz})$ and variations of air pressure $(0.8 \mathrm{bar} / 1.5 \mathrm{bar})$ and amplitudes $(0.5 \mathrm{~mm} / 0.7 \mathrm{~mm})$. Each participant underwent four stimulation blocks covering all possible combinations of air pressure and amplitude conditions (four conditions). The order of the simulation blocks was fixed across participants. Each stimulation block included three runs of $30 \mathrm{~s}$ duration for each frequency condition $(0 \mathrm{~Hz} / 20 \mathrm{~Hz} / 80 \mathrm{~Hz}$, randomized across stimulation blocks) with alternating stimulation (vibration $\mathrm{ON}, 5 \mathrm{~s}$ ) and rest phases (vibration OFF, $5 \mathrm{~s}$ ). Each run was repeated once. The total duration of the postural sway assessment was approximately 30 minutes with a 5 min break in between to relax the legs.

\subsubsection{Postural sway analysis}

The assessment of postural sway in the anterior-posterior direction was based on the raw force plate data sampled at a temporal resolution of $1000 \mathrm{~Hz}$ using the formula $\mathrm{COP}=\mathrm{Mx} / \mathrm{Fz}$ (Brumagne et al., 2004, 2008) and was calculated for each run (spanning vibration and rest phases) within each stimulation block per subject. This resulted in an absolute cumulative distance measure (path length, in $\mathrm{mm}$ ) for each combination of frequency, air pressure and amplitude (12 combinations per subject and per repetition).

JAMOVI software including the GAMLj module was used to analyzed postural sway data (www.jamovi.org). Mixed models were used (generalized mixed models, in case of nonnormally distributed data) with a random factor "Subject" and fixed factors "Frequency(3)" "Repetition(2)", "Air pressure(2)" and "Amplitude(2)". Non-normality of the data was tested using the Shapiro Wilk test and visual inspection of QQ-plots of residuals. Whenever 
appropriate, Bonferroni corrections were applied. Removal of potential outliers was performed using the interquartile range (IQR, 1.5x IQR rule).

\subsection{Neuroimaging methods}

\subsubsection{Participants}

Fifteen healthy volunteers ( 7 female; mean age 29.9 years, SD \pm 5.1 ) were recruited and underwent fMRI scanning. Inclusion/exclusion criteria and ethical approvals were identical to those for the postural sway validation described above (see Section 2.1.3).

\subsubsection{Paradigm}

Prior to the attachment of the pneuVID units, nine different spinous processes (T3, T5, T7, T9, T11, L1, L3, L5 and sacral[S1]) were marked through palpation by an experienced physiotherapist. Twenty pneuVID vibrotactile stimulation units were applied bilaterally to the nine paraspinal locations along the erector spinae muscles (Fig. 1B) and one on the calves of the legs at the mTS, using adhesive tape and a sugar-based glue that was developed in-house. These stimulators were activated, always bilaterally at a single one of the ten locations, at either $20 \mathrm{~Hz}$ or $80 \mathrm{~Hz}$ vibration frequency, in a pseudorandomised order (no more than three occasions in a row at the same location or frequency) that differed per run and per participant. Stimulation events were always of five seconds duration and the inter-stimulation interval was jittered within the range of 4-7 s. A total of 120 stimulation events were delivered per run (12 per location, further divided into 6 per frequency type per location). Two separate runs of the pneuVID stimulation paradigm were delivered to each participant during fMRI data acquisition. Prior to 
the experimental runs, all participants underwent a descending test vibration sequence where they consistently reported feeling stimulation at each location in the midline of the back (i.e., they perceived no lateralisation of stimulation) and at the mTS and could distinguish perceptions evoked by $80 \mathrm{~Hz}$ and $20 \mathrm{~Hz}$ at each of these locations.

\subsubsection{Image acquisition}

Neuroimaging data were acquired using a 3 T Philips Achieva MRI scanner with a 32-channel sensitivity encoding (SENSE) head coil (Philips, Best, The Netherlands) at the MRI Center of the Psychiatric Hospital, University of Zurich (PUK). The stimulation paradigm was separated into two runs of 22.5 minutes length each. We acquired 750 volumes of T2*-weighted echoplanar imaging (EPI) data sensitive to blood-oxygenation level-dependent (BOLD) contrast during both paradigm runs $\left(\mathrm{TR}=1.8 \mathrm{~s} ; \mathrm{TE}=34 \mathrm{~ms}\right.$; flip angle $=70^{\circ} ; 54$ interleaved ascending axial slices with multiband factor $=3$; in-plane resolution $=1.72 \times 1.72 \mathrm{~mm}$; slice thickness $=$ $2.0 \mathrm{~mm}$ with $0 \mathrm{~mm}$ slice gap; SENSE factor $=1.4$; EPI factor $=87$ ). The scanner automatically acquired and removed initial dummy volumes for magnetic equilibration. Before the second stimulation run, we also acquired a high-resolution anatomical T1-weighted (T1w) volume $\left(\mathrm{MPRAGE} ; \mathrm{TR}=6.6 \mathrm{~ms} ; \mathrm{TE}=3.1 \mathrm{~ms} ;\right.$ flip angle $=9^{\circ}$; field-of view $230 \times 226 \times 274$; voxel size $=1.0 \times 1.0 \times 1.2 \mathrm{~mm}$; turbo field echo factor $=203)$.

\subsubsection{Image preprocessing}

Prior to preprocessing, the neuroimaging data were converted to the consensus Brain Imaging Data Structure (BIDS) format (Gorgolewski et al., 2016) and then subjected to preliminary assessment using the FMRIprep pipeline (v. 20.2.0) (Esteban et al., 2018). As part of this procedure, each $\mathrm{T} 1 \mathrm{w}$ volume was corrected for intensity non-uniformity and skull-stripped 
(using the OASIS template) using Advanced Normalization Tools (ANTs) v2.1.0 (Tustison et al., 2010). Brain surfaces were reconstructed using recon-all from FreeSurfer v6.0.1 (Dale et al., 1999), and the brain mask estimated previously was refined with a custom variation of the method to reconcile ANTs-derived and FreeSurfer-derived segmentations of the cortical graymatter of Mindboggle (Klein et al., 2017). Spatial normalization to the ICBM 152 Nonlinear Asymmetrical $(\mathrm{MNI})$ template $6^{\text {th }}$ generation version was performed through nonlinear registration with ANTs v2.1.0 (Avants et al., 2008), using brain-extracted versions of both T1w volume and template. Brain tissue segmentation of cerebrospinal fluid (CSF), white-matter (WM) and gray-matter (GM) was performed on the brain-extracted T1w using FSL (v5.0.9) FAST (Zhang et al., 2001).

Functional data were motion corrected using the MCFLIRT tool of FSL (Smith et al., 2004). This was followed by co-registration with six degrees of freedom to the corresponding T1w image using boundary-based registration (Greve and Fischl, 2009). Motion correcting transformations, BOLD-to-T1w transformation and T1w-to-template (MNI) warp were concatenated and applied in a single step using ANTs v2.1.0 (using Lanczos interpolation).

We selected mean time series, their squared versions and the derivatives thereof from CSF and WM tissue sources, as calculated by FMRIprep, for use as confounding signals with which to filter the fMRI data $(\mathrm{NaN}$ values at the beginning of the derivative vectors were instead computed as the mean value of the signal vector, in order to allow for regression). In addition to filtering for these eight confound vectors, we also regressed out the time series from all components in the data identified as (primarily motion) artefacts by ICA-based Automatic Removal Of Motion Artifacts (ICA-AROMA; Pruim et al., 2015). This regression filtering of tissue-based and ICA-based identified artefactual noise was performed in a single step, without 
any temporal filtering (of the data or the confound regressors), on the unsmoothed, MNI-spacenormalized output of FMRIprep, prior to any participant-level statistical analyses, using $f_{s}{ }_{-}$regfilt. The outputs from this step thus comprised "aggressively denoised" (Pruim et al., 2015) fMRI datasets from the stimulation paradigm runs.

\subsubsection{Statistical analysis}

The denoised datasets from the previous processing stage were analysed per run at the first/participant-level using FSL FEAT (Version 6.00) with FILM prewhitening enabled (Smith et al., 2004). For this initial analysis step, a high pass temporal filter (100 s) was applied to both the fMRI data and the simulation paradigm model design, while the data were also smoothed using a $4 \mathrm{~mm}$ full width at half maximum (FWHM) Gaussian kernel. Base regressors for each of the 20 stimulation settings (10 locations $\times$ two frequency settings) were modelled as separate explanatory variables (EVs). These 20 EVs were defined per run in terms of the precise onsets of each of the six stimulation events per frequency and location for a given setting, with their durations (5 s) fully modelled, using a haemodynamic response function with Gamma convolution. General linear model (GLM) contrasts of interest were computed including comparisons of: (i) $80 \mathrm{~Hz}>20 \mathrm{~Hz}$ across all nine paraspinal locations (the mTS leg units were omitted); (ii) the inverse $20>80 \mathrm{~Hz}$ contrast; (iii) frequency-specific contrasts of the upper four pneuVID units (T3, T5, T7, T9) > lower four panels (L1, L3, L5 and sacral[S1]); and (iv) the inverse 4 lower $>4$ upper contrast. In addition, we examined contrasts for individual EV (location per frequency) representations, in order to explore potentially more fine-grained neural representations, in particular of proprioceptive input. 
Outputs from first-level analyses were subjected to a second, mid-level analysis at the level of individual participants, wherein contrast parameter estimates from the two runs of each participant were tested for their mean representation at the level of fixed effects (using FSL FEAT).

Finally, the resulting averaged participant-level contrasts of interest were entered into mixed effects-level one-sample $t$-tests at the group level using FSL FEAT. The resulting statistical maps of group-level parametric effects for the four contrasts of interest described above were thresholded for significance using whole-brain, cluster-level $(Z>2.3$; p $<0.05)$ correction for testing across multiple voxels.

\section{Results}

\subsection{Postural sway}

QQ-plots of the residuals and the Shapiro-Wilk test indicated non-normality (left-skewed) of the postural sway data $(\mathrm{p}<0.001)$. Furthermore, the Akaike information criterion (AIC) suggested a gamma distribution as the most appropriate type of probability distribution. Therefore, a generalized mixed model with gamma distribution was used. Ten datapoints were removed in the outlier analysis. No significant interaction effects were detected (all $p>0.159)$ and subsequently removed from the model. The fixed effects analysis indicated a significant main effect for "Frequency" $\left(\mathrm{X}^{2}=6.905, \mathrm{df}=2, \mathrm{p}=0.032\right.$ / removal of outliers: $\left.\mathrm{X}^{2}=8.29, \mathrm{df}=2, \mathrm{p}=0.016\right)$ and "Amplitude" $\left(\mathrm{X}^{2}=13.511, \mathrm{df}=1, \mathrm{p}=0.001 /\right.$ removal of outliers: $\mathrm{X}^{2}=18.03, \mathrm{df}=1, \mathrm{p}=$ 0.001), but no significant main effects for "Air pressure" and "Repetition" (all $\mathrm{p}>0.5$ with and 
without outliers). Post-hoc tests indicated a significant difference in postural sway (Fig. 2) between the $80 \mathrm{~Hz}$ (mean $=314 \mathrm{~mm}, \mathrm{SD} \pm 98.6 \mathrm{~mm}$; removal of outliers: mean $=307 \mathrm{~mm}$, $\mathrm{SD} \pm 86.5 \mathrm{~mm})$ and $20 \mathrm{~Hz}($ mean $=301 \mathrm{~mm}, \mathrm{SD} \pm 108 \mathrm{~mm}$; removal of outliers: mean $=291 \mathrm{~mm}$ $\mathrm{SD} \pm 88.3 \mathrm{~mm})$ conditions $\left(\mathrm{z}=2.4\right.$, pbonferroni $=0.049 /$ removal of outliers: $\mathrm{z}=2.55$, $\mathrm{p}_{\text {bonferroni }}=$ $0.032)$ as well as between the $80 \mathrm{~Hz}$ and $0 \mathrm{~Hz}$ (mean $=303 \mathrm{~mm}, \mathrm{SD} \pm 107 \mathrm{~mm}$; removal of outliers: mean $=291 \mathrm{~mm}, \mathrm{SD} \pm 87.0 \mathrm{~mm})$ conditions $(\mathrm{z}=2.18$, puncorrected $=0.030$, pbonferroni $=$ 0.089 / removal of outliers: $z=2.48$, pbonferroni $=0.039$ ). The comparison between $20 \mathrm{~Hz}$ and $0 \mathrm{~Hz}$ condition was not significant (both $\mathrm{p}>0.812$ with and without outliers). Stimulating with an amplitude of $0.7 \mathrm{~mm}$ (mean $=315 \mathrm{~mm}, \mathrm{SD} \pm 97.2 \mathrm{~mm}$; removal of outliers: mean $=310 \mathrm{~mm}$, $\mathrm{SD} \pm 89.2 \mathrm{~mm})$ led to a significant increase in postural sway $(\mathrm{z}=3.68$, ponferroni $=0.001$, removal of outliers: $\mathrm{z}=4.25$, pbonferroni $=0.001)$ compared to $0.5 \mathrm{~mm}($ mean $=297 \mathrm{~mm}, \mathrm{SD} \pm 111 \mathrm{~mm}$; removal of outliers: mean $=283 \mathrm{~mm}, \mathrm{SD} \pm 83.2 \mathrm{~mm}$ ).

\subsection{Neuroimaging results}

We found BOLD fMRI activation to be significantly increased under $80 \mathrm{~Hz}$, relative to $20 \mathrm{~Hz}$ vibrotactile stimulation of the back in several brain regions (Fig. 3; Table 1). These significant clusters were located in bilateral primary somatosensory and motor cortical regions, as well as right supramarginal gyrus extending into posterior insula, secondary somatosensory cortex and temporoparietal junction regions. More fine-grained spatial analysis of these three activation clusters located in motor and somatosensory cortical regions that were central to our hypotheses, revealed the peak activations to be located, based on their correspondence with the Juelich Histological Atlas (Geyer et al., 1996, 2000): with highest probability for Cluster "Index 1" (Table 1) in the left primary somatosensory cortex S1 (region 3a $29 \%$, with $10 \%$ probability of location in S1 3b) and $15 \%$ in primary motor cortex M1 4p; for Cluster Index 2 in right S1 3a 
(67\%, with $10 \%$ probability of location in $\mathrm{S} 13 \mathrm{~b}$ ) and $18 \%$ in $\mathrm{M} 1$ posterior; and for Cluster Index 3 in right posterior supramarginal gyrus $(11 \%)$. More detailed cluster information is provided in Table 1.

In addition to greater activation observed under high frequency stimulation, we also found some brain regions to be significantly more activated by the lower frequency $20 \mathrm{~Hz}$, relative to $80 \mathrm{~Hz}$, stimulation (Fig. 3A; Table 2). These cluster peaks were located, firstly, at the midline in bilateral primary motor and somatosensory cortex, specifically in M1 anterior ( $85 \%$, with only $20 \%$ probability of location in M1 posterior) and, secondly and thirdly, bilaterally in primary somatosensory cortex, predominantly in S1 3b (left $43 \%$, right 41\%). More detailed cluster information is provided in Table 2.

Overall, these described frequency-dependent results appeared to pursue a pattern of differential representations in key sub-regions of both S1 and M1, seemingly in an opposing manner for the two frequency contrasts. Therefore, we further amalgamated the atlas-based probabilistic information of the relevant cluster activation peaks (i.e., Table 1 Cluster Indices 1 and 2; Table 2 Cluster Indices 1-3) in order to permit a simple graphical representation of how the sub-regional dominances of S1 3a vs 3b and M1 anterior vs posterior differed across stimulation frequencies (Fig. 3B).

Finally, we tested for differences between cortical representations of the thoracic (upper) and lumbar including sacral[S1] (lower) paraspinal stimulation, while also exploring more finegrained representational variability along the thoracolumbar axis. We found significant differences between thoracic and lumbar cortical representations under vibrotactile stimulation at $80 \mathrm{~Hz}$, but not at $20 \mathrm{~Hz}$ (Fig. 4). Specifically, at $80 \mathrm{~Hz}$ we found greater activation to upper, 
relative to lower back stimulation, in the right inferior parietal cortex extending into the superior temporal sulcus, as well as in bilateral regions of primary somatosensory cortex (Table 3). Atlas comparisons revealed the peak activations of these latter clusters to be located in regions of high probabilistic overlap between multiple S1 sub-regions, with the left hemisphere result slightly favouring S1 3b (40\%, with 38\% probability for S1 3a and 29\% for Brodmann Area [BA] 2) and the right hemisphere somewhat favouring BA2 (38\%, with 33\% for S1 3a and 29\% for S1 3b). For the inverse contrast ( $80 \mathrm{~Hz}$ : lumbar $>$ thoracic), however, a single significant cluster located with strong probability $(76 \%)$ in the right superior parietal lobule was detected (Table 3 ; an equivalent effect in the left hemisphere did not reach the cluster thresholding level required for statistical significance). No significant differences were identified for either of these contrasts in the $20 \mathrm{~Hz}$ stimulation condition. The results from our exploratory analysis of individual stimulation locations revealed some evidence of a representational progression along the thoracolumbar axis of primary somatosensory cortex (Fig. 5), where peak statistical activations and local maxima (see Supplementary Materials) tended to be represented in more ventral and anterior regions under upper back (thoracic) stimulation and in more dorsal and posterior regions under lower back (lumbar) stimulation.

\section{Discussion}

We present a first behavioural and neurobiological validation of the novel 'pneuVID' method for stimulating muscle spindles and mapping cortical representations of paraspinal afferent inputs. In particular we sought to delineate the neural representations of proprioceptive stimulation associated with the activation of paraspinal muscle spindles (and thus the preparation of motor 
systems), relative to tactile stimulation. Additionally, we attempted to provide an initial indication of the cortical somatotopy of multiple paraspinal locations across the thoracolumbar axis. The results suggest a spatial delineation of the functional representations of both paraspinal proprioceptive/tactile and upper/lower back sensory input across the span of the primary somatosensory and, to some extent, primary motor cortex. The pneuVID methodology may therefore serve as a promising tool for future investigation of alterations in the cortical maps of paraspinal input and their role in the persistence of LBP.

Firstly, we found distinct patterns of cortical activation representing high-frequency $(80 \mathrm{~Hz})$ 'proprioceptive' and low-frequency $(20 \mathrm{~Hz})$ 'tactile' vibratory stimulations using pneuVID. In $\mathrm{S} 1$, peak proprioceptive representations were localised with a higher probability in region S1 3a (on average 47\%; S1 3b=10\%), while tactile activation was pinpointed to a greater extent in $\mathrm{S} 1$ 3b (average 28\%; S1 3a $<1 \%$ ). This observation supports our hypothesis of spatial differences between cortical representations of tactile and proprioceptive sensory information and is in line with prior findings detailed in the primate literature regarding the modality-specificity of equivalent hand and limb somatosensory perception (for review see, e.g., Delhaye et al., 2018); although see also (Kim et al., 2015). In addition, these peak activations were probabilistically localised more in primary motor cortex region M1 4p under proprioceptive stimulation (average 16.5\%; M1 4a < 1\%) and in M1 4a $(40.3 \%$; M1 4p = 8.3\%) under tactile stimulation. These findings of somatosensory information processing in motor cortical areas are in line with prior human and animal evidence in the literature, as it is known both that M1 activation is necessary for the somatosensory experience associated with movement of body parts such as the hand (Naito et al., 2002) and that cutaneous and proprioceptive inputs have direct pathways to M1 mediated through the spinothalamic and dorsal column tract, respectively (Asanuma et al., 1980; 
Avanzino et al., 2014). Furthermore, the observations in M1 appear to follow on from those in $\mathrm{S} 1$ as region $4 \mathrm{p}$, also known as "New M1", is understood to be a phylogenetically newer evolutionary manifestation than region 4a ("Old M1"), with the former being identified as specifically attuned to the complex processing of skilled motor behaviours characteristic of higher-order primates due to the prevalence of direct, monosynaptic connections between cortico-motor cells and muscle-adjacent motor neurons (Rathelot and Strick, 2009). This can be interpreted as mirroring the observed dissociations in $\mathrm{S} 1$ as area $3 \mathrm{a}$, which displayed a vibrotactile stimulation-related activation pattern more similar to $\mathrm{M} 14 \mathrm{p}$ than $4 \mathrm{a}$, is also understood to be a phylogenetically newer region than S1 3b (Kaas, 2004). It is reasonable to assume that such cross-modal evolutionary development, integrating proprioceptive processing within newer sub-regions of somatosensory and motor cortex, has contributed to the amplification of complex abilities in humans and other higher order primates. In the current work we extend this framework of cross-modal integration to suggest that it applies to the human back and not only to bodily regions typically associated with more complex motor control and somatosensory perception, such as the hand. Further support for an increased complexity of the representations of spinal sensorimotor control in higher-order cortical regions has previously been provided by transcranial magnetic stimulation evidence of distinct motor cortical locations being associated with the excitation of deeper, relative to more superficial, paraspinal muscle fibres at the same lumbar location, again underlining the human ability for highly finely-graded control of these muscles (Tsao et al., 2011a). Interestingly, this complexity is thought to be central to our ability to adapt our movements and perceptions to the experience of pain (Hodges and Tucker, 2011; Tsao et al., 2011b; Elgueta-Cancino et al., 2021). 
Secondly, we have expanded upon the work pioneered by Penfield (Penfield, 1947) and continued by others (e.g., Boendermaker et al., 2014; Roux et al., 2018) in mapping the functional topography of human somatosensory cortex, by providing additional information about fine-grained somatotopy of the back. Previously, very little has been established regarding the functional spatial organization of S1 with respect to the proprioceptive and other somatosensory representations of the back. In brief, Penfield first identified representational locations of the hip and the shoulder on the convexity of the postcentral gyrus and drew the representation of the back between these two areas (Penfield, 1947), while, more recently, intracortical stimulation of BA1 in S1 has identified the representations of the thorax and abdomen to indeed lie between the hip and the shoulder (Roux et al., 2018), although the cortical somatotopic representation of the back along the thoracolumbar axis, and in particular of proprioceptive afferents, has remained unclear. Interestingly, a shifted representation of tactile stimuli applied to the back, away from the typically more lateral S1 regions and towards the midline, has been observed using magnetencephalography (MEG) in a small group of chronic LBP patients (Flor et al., 1997). In the current study, using fMRI, we were able to image S1 activation and other relevant brain regions at a higher spatial resolution, including information about cortical targets of different sensory afferents from different paraspinal locations and thereby providing a promising basis for further investigations in chronic LBP patients. At a coarse, but statistically highly-powered level, we found proprioceptive $(80 \mathrm{~Hz})$ stimulation of the upper back, including thoracic areas, to activate regions of the inferior parietal cortex (angular and supramarginal gyri) and the anterior primary somatosensory cortex (S1 BA2, 3a and 3b), while lower back stimulation including lumbar areas activated more superior and posterior regions of parietal cortex (superior parietal lobule area "5L"). At a finer-grained - but ultimately less statistically 
well-powered - level, when considering neuronal activations to stimulation of individual bilateral paraspinal locations, we have further confirmed this directional somatotopy during proprioceptive stimulation (see Fig. 5 and Supplementary Materials). Regarding the apparent right hemisphere lateralisation of these above somatotopic observations, the present data provide limited scope with which to speculate about a hemispheric specialisation of function, given the fairly modest sample size. However, a precedent for this finding has perhaps previously been set in terms of highlighting the dominance of the right hemisphere in the perception of limb movement (Naito et al., 2005), a phenomenon that we contend may generalise to other areas of the somatosensory homunculus, including the back. In addition, it is worth noting that we were only able to explore and discuss differential somatotopic representations for $80 \mathrm{~Hz}$ stimulation representations in detail, as for the majority of single-location $20 \mathrm{~Hz}$ stimulation conditions no significant clusters were identified (in the $80 \mathrm{~Hz}$ condition stimulation of each paraspinal location was associated with at least one significant cluster). This is likely to be a corollary of the small sample size, which should be expanded upon in future neuroimaging studies attempting to investigate fine-grained tactile-perceptual representations of the back.

Further to the limitations already mentioned, it is important to discuss the possibility that our purported proprioception-specific fMRI findings might instead specifically represent processes of differentiation between and encoding of (high and low) vibrotactile stimulation frequencies. Unfortunately, as we stimulated nine paraspinal locations at just two separate frequencies, in order to elevate statistical power for each condition without extending scan time to an uncomfortable length for participants, we are unable to test the frequency-dependence of representations in a more directly parametric manner using the current data. However, prior evidence has revealed that frequency-dependent representations of vibrotactile stimulation of the 
human index finger are more densely located in the lateral sulcus of S2 than in S1 or other regions of S2 (Chung et al., 2013), or in highly lateral regions of somatosensory cortex (reported as S1 but with peak coordinates located most probabilistically in S2 and the inferior parietal lobule) extending into the supramarginal gyrus (Kim et al., 2016). Therefore, it is possible that our findings, which centred around (comparatively medial regions of) S1, are more indicative of tactile/proprioceptive afferent differentiation than of simple frequency encoding differences. Either way, it is likely that the differences between cellular processes distinguishing tactile/proprioceptive and frequency-encoding representations in S1 are too microstructural and interwoven in nature to be detected using whole-brain fMRI. Instead, it may only be currently feasible to delineate these processes using direct electrical recording techniques of small populations of neurons, above the limits of spatial resolution presently achievable with fMRI. However, progress in this area with fMRI may be expedited using computationally-inspired models of mechanistic spatial or frequency representation, for example with multi-voxel pattern analysis such as representational similarity analysis (Kim et al., 2016). In addition, higher field (e.g., 7 Tesla) MRI acquisitions may enable increased spatial delineation of different mechanistic processes due to the higher spatial resolutions they are able to achieve with fMRI, including at the level of different cortical layers: so-called "laminar fMRI" (Lawrence et al., 2019; Stephan et al., 2019).

Finally, it is not fully clear which specific trunk muscle spindles are targeted by vibratory stimulation with pneuVID. The primary assumption is that superficial (longissimus and spinalis) muscles along the thoracolumbar axis are most affected in terms of their activation. However, 80 $\mathrm{Hz}$ pneuVID stimulation might also penetrate to deeper muscles such as the rotatores and 
multifidi muscles, which are particularly dense in muscle spindle fibres and therefore also involved in key aspects of trunk and back proprioception (Boucher et al., 2015).

\section{Conclusion}

In sum, our findings highlight the potential for tools such as pneuVID to significantly extend previous work on mapping the cortical representation of paraspinal sensory input. Future combinations of the pneuVID technique with advanced computational and imaging methods should pave the way for new opportunities to elucidate a potential causal role of cortical reorganization processes in chronic LBP. Ultimately, this might provide promising neuroimaging-based outcomes with which to test the potential therapeutic effect of individualized therapies such as motor control exercises and how they compare to other treatment approaches (e.g., neuromodulation).

\section{Competing interests}

No competing interests were disclosed.

\section{Acknowledgments}

We thank Magdalena Suter for help with data collection and recruitment of participants. This research was supported by the Swiss National Science Foundation (SNF, Bern, Switzerland). 


\section{Tables}

Table 1. Brain regions significantly more activated under $80 \mathrm{~Hz}$, relative to $20 \mathrm{~Hz}$, vibrotactile stimulation of the back. Three regional statistical maps displayed significantly greater activation (cluster $Z>2.3 ; \mathrm{p}<0.05$ ) during $80 \mathrm{~Hz}$ stimulation than during $20 \mathrm{~Hz}$ simulation. Statistical and anatomical co-ordinate information (relative to the MNI152 standard space template) is provided. $\mathrm{R}=$ right hemisphere; $\mathrm{L}=$ left hemisphere; $\mathrm{M} 1$ = primary motor cortex; S1 = primary somatosensory cortex; $\mathrm{S} 2=$ secondary somatosensory cortex; SPL = superior parietal lobule; TPJ = temporoparietal junction; BA = Brodmann area.

\begin{tabular}{|c|c|c|c|c|c|c|c|}
\hline $\begin{array}{l}\text { Cluster } \\
\text { Index }\end{array}$ & Voxels & $\mathbf{P}$ & $\begin{array}{l}Z- \\
\max \end{array}$ & $\begin{array}{l}x \\
(\mathrm{~mm})\end{array}$ & $\begin{array}{l}y \\
(\mathrm{~mm})\end{array}$ & $\begin{array}{l}z \\
(\mathrm{~mm})\end{array}$ & Anatomical location \\
\hline 1 & 241 & $\begin{array}{l}< \\
0.001\end{array}$ & 3.89 & -16 & -36 & 52 & L S1 BA3a \& 3b/M1 4p/SPL \\
\hline 2 & 191 & $\begin{array}{l}< \\
0.001\end{array}$ & 3.85 & 16 & -36 & 54 & R S1 BA3a \& 3b/M1 4p/SPL \\
\hline 3 & 111 & 0.040 & 3.68 & 44 & -38 & 14 & $\begin{array}{l}\text { R supramarginal } \\
\text { gyrus/S2/TPJ/posterior insula }\end{array}$ \\
\hline
\end{tabular}


Table 2. Brain regions significantly more activated under $20 \mathrm{~Hz}$, relative to $80 \mathrm{~Hz}$, vibrotactile stimulation of the back. Three regional statistical maps displayed significantly greater activation (cluster $Z>2.3 ; \mathrm{p}<0.05$ ) during $20 \mathrm{~Hz}$ stimulation than during $80 \mathrm{~Hz}$ simulation. Statistical and anatomical co-ordinate information (relative to the MNI152 standard space template) is provided. $\mathrm{R}=$ right hemisphere; $\mathrm{L}=$ left hemisphere; $\mathrm{M} 1=$ primary motor cortex; S1 = primary somatosensory cortex; $\mathrm{SPL}=$ superior parietal lobule; $\mathrm{BA}=$ Brodmann area.

\begin{tabular}{|c|c|c|c|c|c|c|c|}
\hline $\begin{array}{l}\text { Cluster } \\
\text { Index }\end{array}$ & Voxels & $\mathbf{P}$ & $\begin{array}{l}Z- \\
\max \end{array}$ & $\begin{array}{l}x \\
(\mathrm{~mm})\end{array}$ & $\begin{array}{l}y \\
(\mathrm{~mm})\end{array}$ & $\begin{array}{l}z \\
(\mathrm{~mm})\end{array}$ & Anatomical location \\
\hline 1 & 293 & $\begin{array}{l}< \\
0.001\end{array}$ & 3.45 & -6 & -30 & 70 & $\begin{array}{l}\text { Bilateral M1 4a/S1 } \\
\text { BA3b/SPL }\end{array}$ \\
\hline 2 & 127 & 0.017 & 3.32 & 62 & 0 & 28 & $\begin{array}{l}\text { R S1 BA3b \& } \\
\text { BA1/BA6/M1 4a }\end{array}$ \\
\hline 3 & 114 & 0.045 & 3.52 & -58 & -12 & 36 & $\begin{array}{l}\text { L S1 BA3b \& } \\
\text { BA2/BA1/M1 4a }\end{array}$ \\
\hline
\end{tabular}


Table 3. Brain regions significantly more activated under thoracic, relative to lumbar, 80

Hz proprioceptive vibrotactile stimulation of the back and vice versa. Three regional statistical maps displayed significantly greater activation (cluster $Z>2.3$; p $<0.05$ ) during thoracic stimulation than during lumbar simulation, while one showed the opposite effect. Statistical and anatomical co-ordinate information (relative to the MNI152 standard space template) is provided. $\mathrm{Th}=$ thoracic; $\mathrm{Lu}=$ lumbar; $\mathrm{R}=$ right hemisphere; $\mathrm{L}=$ left hemisphere; $\mathrm{M} 1$ = primary motor cortex; $\mathrm{S} 1$ = primary somatosensory cortex; SPL = superior parietal lobule; $\mathrm{STS}=$ superior temporal sulcus; BA = Brodmann area.

\begin{tabular}{|c|c|c|c|c|c|c|c|}
\hline Contrast & Voxels & $\mathbf{P}$ & $\begin{array}{l}Z- \\
\max \end{array}$ & $\begin{array}{l}x \\
(\mathrm{~mm})\end{array}$ & $\begin{array}{l}y \\
(\mathrm{~mm})\end{array}$ & $\begin{array}{l}z \\
(\mathrm{~mm})\end{array}$ & Anatomical location \\
\hline \multirow[t]{5}{*}{$\mathrm{Th}>\mathrm{Lu}$} & 200 & $<0.001$ & 3.26 & 64 & -46 & 16 & $\begin{array}{l}\mathrm{R} \text { angular \& } \\
\text { supramarginal }\end{array}$ \\
\hline & & & & & & & gyri/STS \\
\hline & 153 & 0.003 & 4.53 & -22 & -36 & 54 & L S1 BA3b \& \\
\hline & & & & & & & $3 \mathrm{~b} / \mathrm{BA} 2$ \\
\hline & 148 & 0.004 & 3.82 & 24 & -34 & 50 & R S1 BA $2 \& 3 a / 3 b$ \\
\hline $\mathrm{Lu}>\mathrm{Th}$ & 165 & 0.002 & 3.61 & 18 & -48 & 70 & R SPL \\
\hline
\end{tabular}




\section{Figure Legends}

Figure 1. (A) Simplified representation of the pneuVID hardware setup (see also Schibli et al., 2021). Via this system, the operator can safely control the vibrotactile stimulation experiment from outside the MRI scanner room. (B) Example image showing placement of pneuVID vibration units on the skin of the back (corresponding, from top to bottom, to the thoracic, lumbar and sacral spinous processes T3, T5, T7, T9, T11, L1, L3, L5 and sacral[S1]).

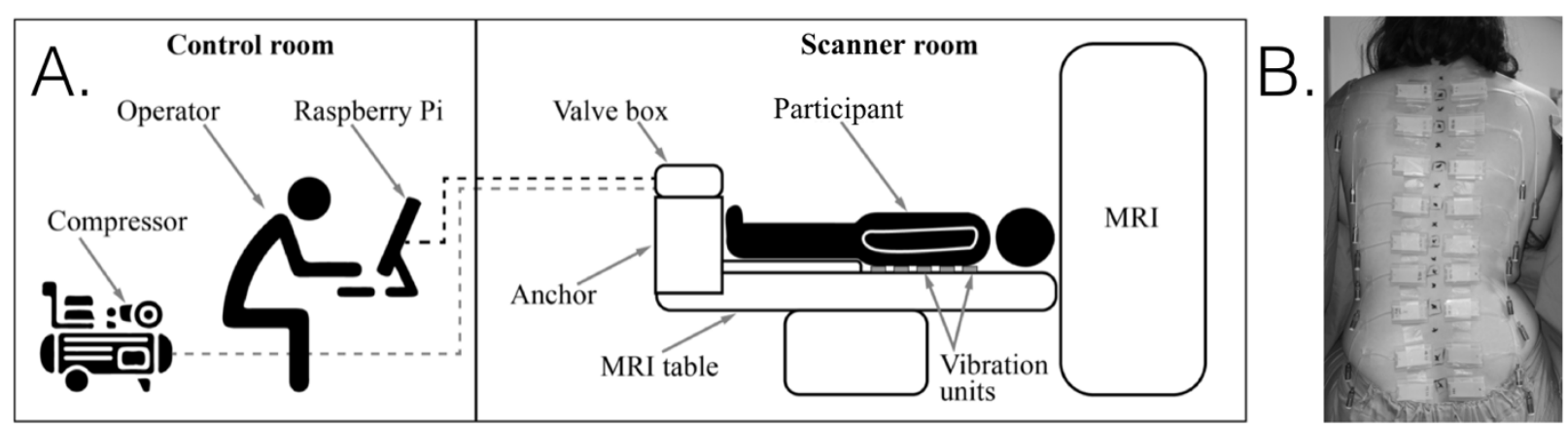


Figure 2. Jittered density plot showing the raw data points (outliers removed). The red dot indicates the median whereas the whiskers indicate the IQR. Significant differences (*) were observed between the $80 \mathrm{~Hz}$ and $20 \mathrm{~Hz}$ conditions $(\mathrm{z}=2.4$, p bonferroni $=0.049 /$ removal of outliers: $\mathrm{z}=2.55$, p bonferroni $=0.032)$ as well as between the $80 \mathrm{~Hz}$ and $0 \mathrm{~Hz}$ conditions $(\mathrm{z}=2.18$, $\mathrm{p}_{\text {uncorrected }}=0.030, \mathrm{p}_{\text {bonferroni }}=0.089 /$ removal of outliers: $\left.\mathrm{z}=2.48, \mathrm{p}_{\text {bonferroni }}=0.039\right)$. The comparison between $20 \mathrm{~Hz}$ and $0 \mathrm{~Hz}$ condition was not significant (both $\mathrm{p}>0.812$ with and without outliers).

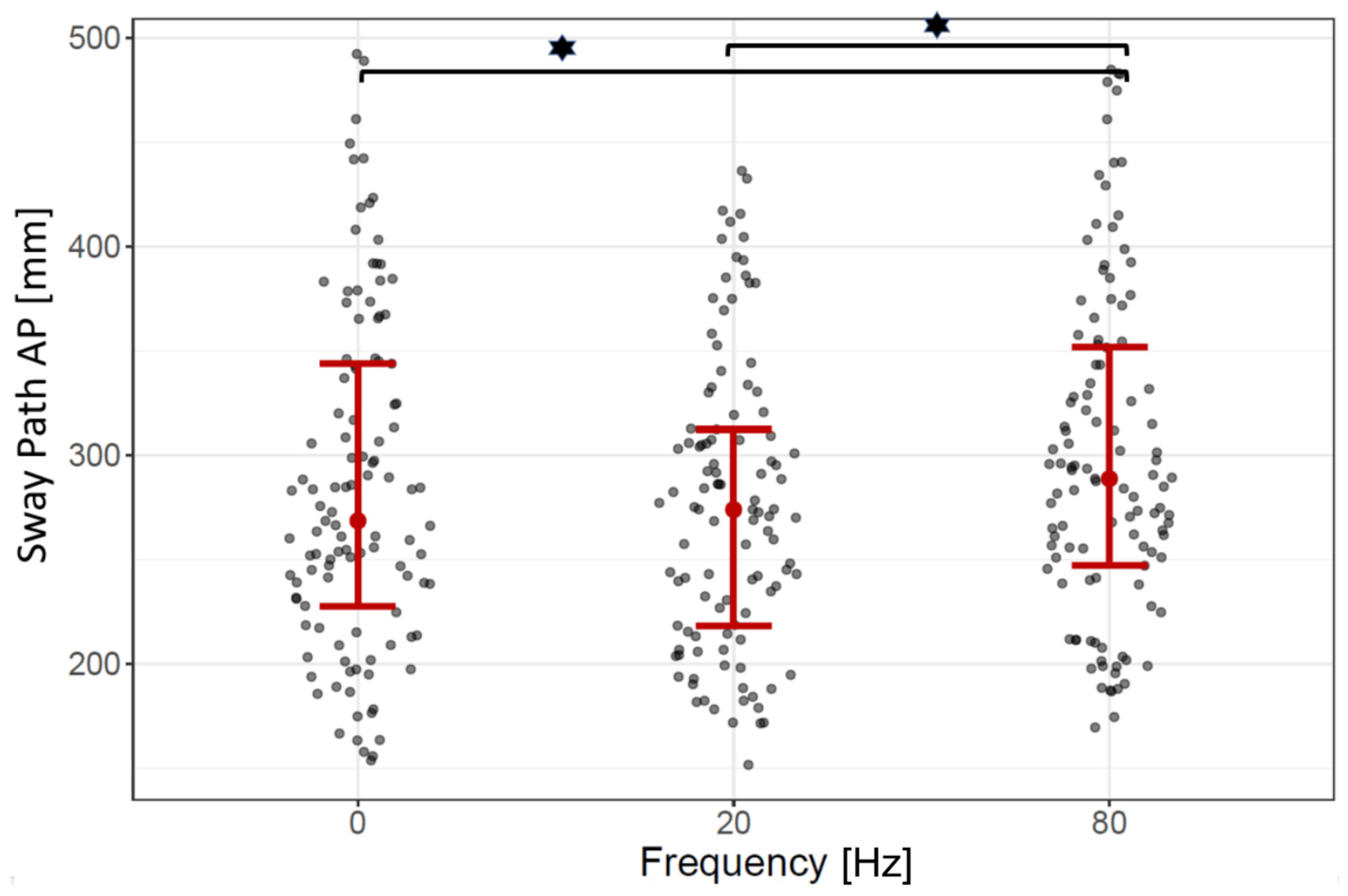


Figure 3. (A) Brain regions displaying differential activation under high and low frequency paraspinal vibrotactile stimulation ( $80 \mathrm{~Hz}$ vs. $20 \mathrm{~Hz}$ ). Red-yellow Z-statistic map and colour bar denote purported proprioception-related $80 \mathrm{~Hz}>20 \mathrm{~Hz}$ significant activation differences (cluster $Z>2.3 ; \mathrm{p}<0.05$ ); blue-cyan denotes (purported non-proprioceptive) $20 \mathrm{~Hz}>80 \mathrm{~Hz}$ tactile stimulation effects. Statistical maps are overlaid on a background image of a $\mathrm{T} 1$ template in MNI152 standard space. $\mathrm{R}=$ right hemisphere; $\mathrm{L}=$ left. NB: axial slices are displayed in line with radiological orientation conventions ( $\mathrm{L} / \mathrm{R}$ hemisphere flipped in figure). (B) Mean \% probability (y-axis) across relevant clusters from (A) spanning regions S1 Area 3 and/or M1 Area 4, in terms of the Juelich Histological Atlas-based likelihood of cluster peaks being located in sub-regions S1 3a, S1 3b, M1 4a and M1 4p; a preferential effect can be seen for proprioception-related stimulation $(80>20 \mathrm{~Hz}$, orange) representation in $\mathrm{S} 13 \mathrm{a}$, relative to $3 \mathrm{~b}$ and in M1 4p, relative to 4a, with an apparent opposing preferential effect for non-proprioceptive tactile stimulation $(20>80 \mathrm{~Hz}$, blue) representation in $\mathrm{M} 14 \mathrm{a}$, relative to $4 \mathrm{p}$ and S1 $3 \mathrm{~b}$, relative to $3 \mathrm{a}$.

A.

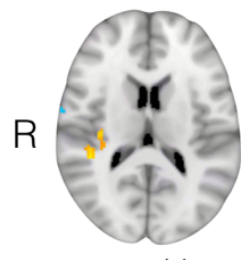

$z=14$

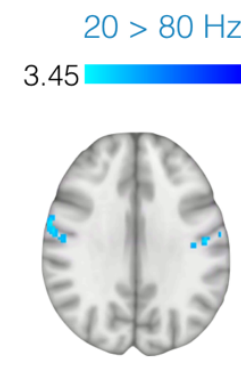

$z=30$
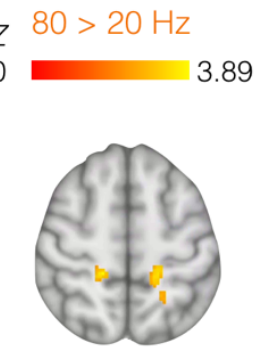

$z=54$

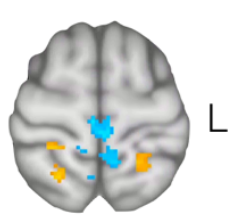

$z=64$

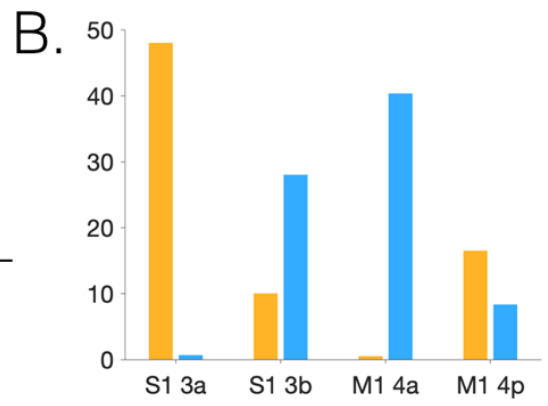


Figure 4. Brain regions displaying differential somatotopy, in terms of functional activation to proprioceptive $(80 \mathrm{~Hz})$ vibrotactile stimulation, depending on the location on the back. Redyellow Z-statistic map and colour bar denote significantly increased activation to thoracic (upper back), relative to lumbar (lower back), stimulation (cluster $Z>2.3$; p $<0.05$ ); blue-cyan denotes a significant inverse (lumbar $>$ thoracic) effect. Statistical maps are overlaid on a background image of a T1 template in MNI152 standard space. $\mathrm{R}=$ right hemisphere.

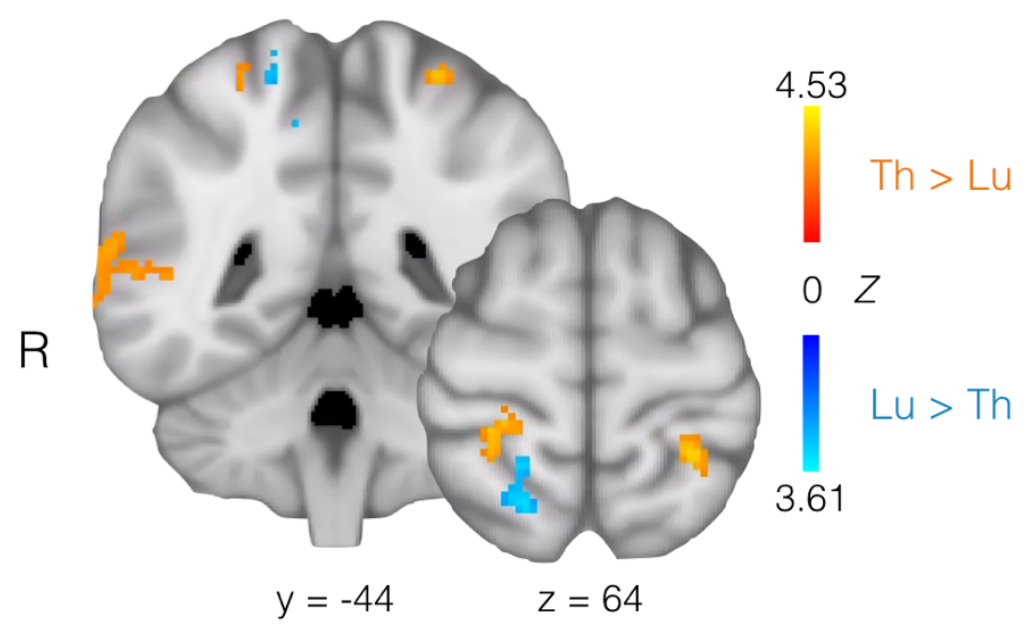


Figure 5. Discrete somatotopy of upper and lower back is visible along the thoracolumbar axis. In the centre diagonal, statistical maps representing activation to $80 \mathrm{~Hz}$ stimulation at the single uppermost (orange-yellow), single precise middle (green) and single lowest (blue) back stimulation points are overlaid, with reduced opacity to aid visualisation of overlap, on multiple sequential slices from a background image of a T1 template in MNI152 standard space, with a focus on the right $(\mathrm{R})$ hemisphere. The activation map representing stimulation of the leg (mTS) is also presented (red) to emphasise the concordance with - and the protraction of - known somatotopy. In the columnar plots, columns represent the frequency with which statistical peaks and other local maxima of activated clusters are located at specific single-plane MNI coordinates in the y-plane (bottom left; $x$-axis denotes anterior-posterior cortical positioning) and the z-plane (top right; $x$-axis = ventral-dorsal cortical elevation) for the uppermost four (orangeyellow), the single middle (green) and the lowest four (blue) stimulation sites; $\left(^{*}\right)$ denotes the planar co-ordinate location of each of the nine peak voxels. The upper back appears to be represented more ventrally and anteriorly and the lower back represented more dorsally and posteriorly in S1 (with the middle back representation positioned in between the two). 
bioRxiv preprint doi: https://doi.org/10.1101/2021.12.19.473341; this version posted December 21, 2021. The copyright holder for this preprint (which was not certified by peer review) is the author/funder, who has granted bioRxiv a license to display the preprint in perpetuity. It is made available under aCC-BY-NC-ND 4.0 International license.

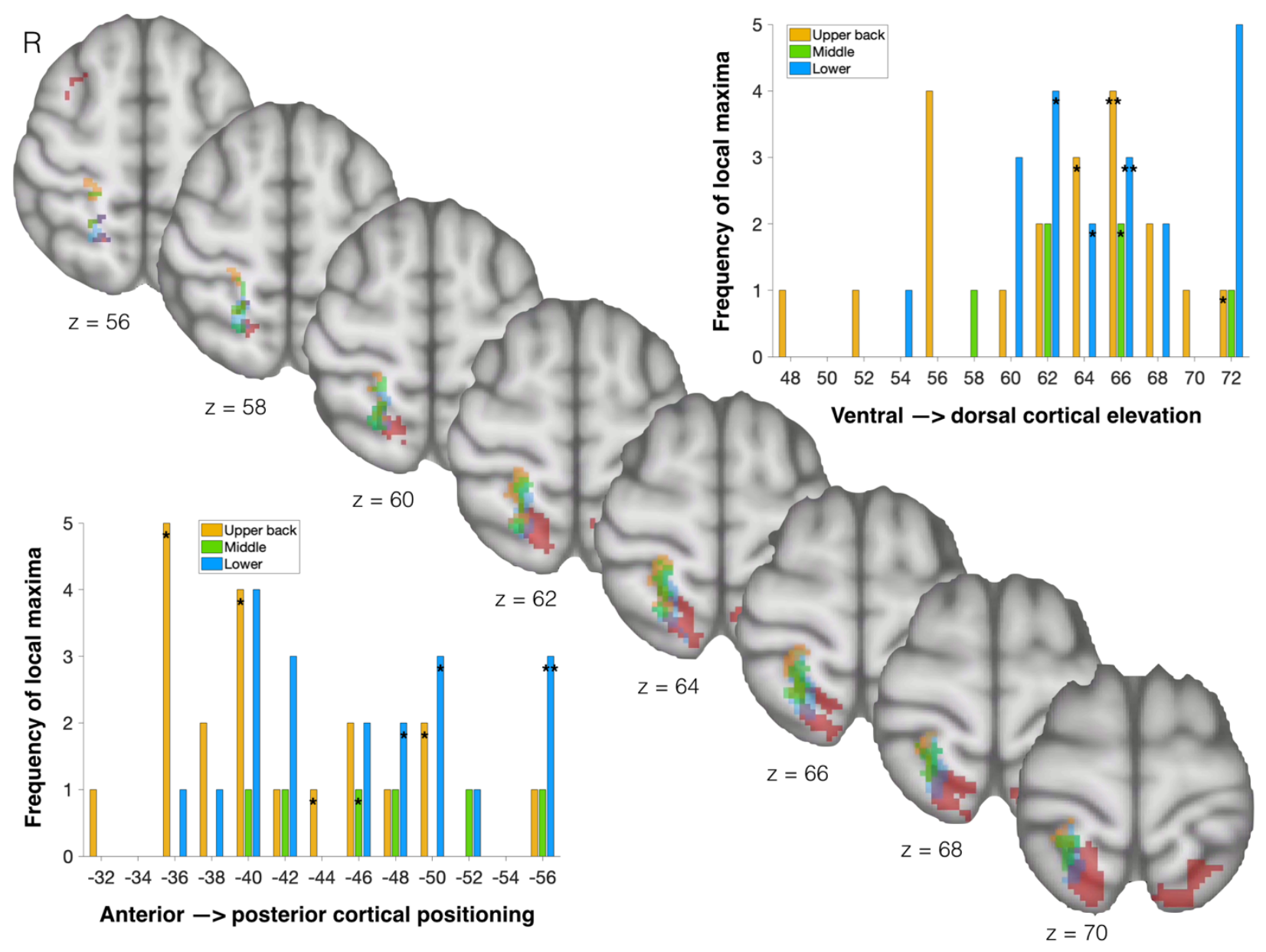




\section{References.}

Apkarian AV, Bushnell MC, Treede RD, Zubieta JK (2005) Human brain mechanisms of pain perception and regulation in health and disease. Eur J Pain 9:463.

Asanuma H, Larsen K, Yumiya H (1980) Peripheral input pathways to the monkey motor cortex. Exp brain Res 38:349-355.

Avants BB, Epstein CL, Grossman M, Gee JC (2008) Symmetric diffeomorphic image registration with cross-correlation: Evaluating automated labeling of elderly and neurodegenerative brain. Med Image Anal 12:26-41.

Avanzino L, Pelosin E, Abbruzzese G, Bassolino M, Pozzo T, Bove M (2014) Shaping Motor Cortex Plasticity Through Proprioception. Cereb Cortex 24:2807-2814.

Beaudette SM, Larson KJ, Larson DJ, Brown SHM (2016) Low back skin sensitivity has minimal impact on active lumbar spine proprioception and stability in healthy adults. Exp Brain Res 234:2215-2226.

Bingel U, Lorenz J, Glauche V, Knab R, Gläscher J, Weiller C, Büchel C (2004) Somatotopic organization of human somatosensory cortices for pain: a single trial fMRI study. Neuroimage 23:224-232.

Boendermaker B, Meier ML, Luechinger R, Humphreys BK, Hotz-Boendermaker S (2014) The cortical and cerebellar representation of the lumbar spine. Hum Brain Mapp 35:3962.

Boucher J-A, Abboud J, Nougarou F, Normand MC, Descarreaux M (2015) The Effects of Vibration and Muscle Fatigue on Trunk Sensorimotor Control in Low Back Pain Patients. PLoS One 10:e0135838.

Brumagne S, Cordo P, Lysens R, Verschueren S, Swinnen S (2000) The role of paraspinal muscle spindles in lumbosacral position sense in individuals with and without low back pain. In: Spine, pp 989-994. Spine (Phila Pa 1976).

Brumagne S, Cordo P, Verschueren S (2004) Proprioceptive weighting changes in persons with low back pain and elderly persons during upright standing. Neurosci Lett 366:63-66.

Brumagne S, Janssens L, Knapen S, Claeys K, Suuden-Johanson E (2008) Persons with recurrent low back pain exhibit a rigid postural control strategy. Eur Spine J 2008 179 17:1177-1184.

Burke D, Hagbarth KE, Löfstedt L, Wallin BG (1976) The responses of human muscle spindle endings to vibration during isometric contraction. J Physiol 261:695-711.

Chung YG, Kim J, Han SW, Kim HS, Choi MH, Chung SC, Park JY, Kim SP (2013) Frequency-dependent patterns of somatosensory cortical responses to vibrotactile stimulation in humans: A fMRI study. Brain Res 1504:47-57.

Claeys K, Brumagne S, Dankaerts W, Kiers H, Janssens L (2010) Decreased variability in postural control strategies in young people with non-specific low back pain is associated with altered proprioceptive reweighting. Eur J Appl Physiol 111:115-123.

Claeys K, Dankaerts W, Janssens L, Pijnenburg M, Goossens N, Brumagne S (2015) Young individuals with a more ankle-steered proprioceptive control strategy may develop mild non-specific low back pain. J Electromyogr Kinesiol 25:329-338.

Dale AM, Fischl B, Sereno MI (1999) Cortical Surface-Based Analysis: I. Segmentation and Surface Reconstruction. Neuroimage 9:179-194.

Delhaye BP, Long KH, Bensmaia SJ (2018) Neural Basis of Touch and Proprioception in Primate Cortex. Compr Physiol 8:1575.

Elgueta-Cancino E, Sheeran L, Salomoni S, Hall | Leanne, Hodges PW, Mazaheri A (2021) 
Characterisation of motor cortex organisation in patients with different presentations of persistent low back pain. Eur J Neurosci https://doi.org/10.1111/ejn.15511.

Esteban O, Markiewicz CJ, Blair RW, Moodie CA, Isik AI, Erramuzpe A, Kent JD, Goncalves M, DuPre E, Snyder M, Oya H, Ghosh SS, Wright J, Durnez J, Poldrack RA, Gorgolewski KJ (2018) fMRIPrep: a robust preprocessing pipeline for functional MRI. Nat Methods 16:111-116.

Flor H (2003) Cortical reorganisation and chronic pain: implications for rehabilitation. J Rehabil Med 41 Suppl:66-72.

Flor H, Braun C, Elbert T, Birbaumer N (1997) Extensive reorganization of primary somatosensory cortex in chronic back pain patients. Neurosci Lett 224:5-8.

Gandevia SC, McCloskey DI, Burke D (1992) Kinaesthetic signals and muscle contraction. Trends Neurosci 15:62-65.

Geyer S, Ledberg A, Schleicher A, Kinomura S, Schormann T, Bürgel U, Klingberg T, Larsson J, Zilles K, Roland PE (1996) Two different areas within the primary motor cortex of man. Nature 382:805-807.

Geyer S, Schormann T, Mohlberg H, Zilles K (2000) Areas 3a, 3b, and 1 of Human Primary Somatosensory Cortex: 2. Spatial Normalization to Standard Anatomical Space. Neuroimage 11:684-696.

Gilman S (2002) Joint position sense and vibration sense: anatomical organisation and assessment. J Neurol Neurosurg Psychiatry 73:473-477.

Goodwin GM, McCloskey DI, Matthews PBC (1972) The contribution of muscle afferents to kinaesthesia shown by vibration induced illusions of movement and by the effects of paralysing joint afferents. Brain 95:705-748.

Gorgolewski KJ et al. (2016) The brain imaging data structure, a format for organizing and describing outputs of neuroimaging experiments. Sci Data 3:160044.

Greve DN, Fischl B (2009) Accurate and robust brain image alignment using boundary-based registration. Neuroimage 48:63-72.

Hodges PW, Cholewicki J, van Dieën JH (2013) Spinal Control: The Rehabilitation of Back Pain: State of the art and science. Churchill Livingstone: London, UK.

Hodges PW, Tucker K (2011) Moving differently in pain: A new theory to explain the adaptation to pain. Pain 152:S90-98.

Jenkins LC, Chang WJ, Buscemi V, Liston M, Skippen P, Cashin AG, McAuley JH, Schabrun SM (2021) Low Somatosensory Cortex Excitability in the Acute Stage of Low Back Pain Causes Chronic Pain. J pain https://doi.org/10.1016/j.jpain.2021.08.003.

Kaas JH (2004) Evolution of somatosensory and motor cortex in primates. Anat Rec Part A Discov Mol Cell Evol Biol 281A:1148-1156.

Kiers H, van Dieën JH, Brumagne S, Vanhees L (2015) Postural sway and integration of proprioceptive signals in subjects with LBP. Hum Mov Sci 39:109-120.

Kim J, Chung YG, Chung SC, Bulthoff HH, Kim SP (2016) Neural Categorization of Vibrotactile Frequency in Flutter and Vibration Stimulations: An fMRI Study. IEEE Trans Haptics 9:455-464.

Kim SS, Gomez-Ramirez M, Thakur PH, Hsiao SS (2015) Multimodal Interactions between Proprioceptive and Cutaneous Signals in Primary Somatosensory Cortex. Neuron 86:555566.

Klein A, Ghosh SS, Bao FS, Giard J, Häme Y, Stavsky E, Lee N, Rossa B, Reuter M, Neto EC, Keshavan A (2017) Mindboggling morphometry of human brains. PLOS Comput Biol 
$13: \mathrm{e} 1005350$.

Langevin HM (2021) Reconnecting the Brain With the Rest of the Body in Musculoskeletal Pain Research. J Pain 22:1-8.

Lawrence SJD, Formisano E, Muckli L, de Lange FP (2019) Laminar fMRI: Applications for cognitive neuroscience. Neuroimage 197:785-791.

Lee AS, Cholewicki J, Reeves NP, Zazulak BT, Mysliwiec LW (2010) Comparison of Trunk Proprioception Between Patients With Low Back Pain and Healthy Controls. Arch Phys Med Rehabil 91:1327-1331.

Lee S, Kruglikov I, Huang ZJ, Fishell G, Rudy B (2013) A disinhibitory circuit mediates motor integration in the somatosensory cortex. Nat Neurosci 16:1662-1670.

Maher C, Underwood M, Buchbinder R (2017) Non-specific low back pain. Lancet 389:736747.

Makin TR, Bensmaia SJ (2017) Stability of Sensory Topographies in Adult Cortex. Trends Cogn Sci 21:195-204.

Massé-Alarie H, Schneider C (2016) Revisiting the Corticomotor Plasticity in Low Back Pain: Challenges and Perspectives. Healthc 2016, Vol 4, Page 67 4:67.

Meier ML, Vrana A, Schweinhardt P (2018) Low Back Pain: The Potential Contribution of Supraspinal Motor Control and Proprioception 25:583-596.

Moseley GL, Flor H (2012) Targeting Cortical Representations in the Treatment of Chronic Pain: A Review 26:646-652.

Naito E, Roland PE, Ehrsson HH (2002) I feel my hand moving: a new role of the primary motor cortex in somatic perception of limb movement. Neuron 36:979-988.

Naito E, Roland PE, Grefkes C, Choi HJ, Eickhoff S, Geyer S, Zilles K, Ehrsson HH (2005) Dominance of the Right Hemisphere and Role of Area 2 in Human Kinesthesia 93:10201034.

Penfield WG (1947) Some observations on the cerebral cortex of man. Proc R Soc London Ser B, Biol Sci 134:329-347.

Proske U, Gandevia SC (2012) The Proprioceptive Senses: Their Roles in Signaling Body Shape, Body Position and Movement, and Muscle Force 92:1651-1697.

Pruim RHR, Mennes M, van Rooij D, Llera A, Buitelaar JK, Beckmann CF (2015) ICAAROMA: A robust ICA-based strategy for removing motion artifacts from fMRI data. Neuroimage 112:267-277.

Rathelot J-A, Strick PL (2009) Subdivisions of primary motor cortex based on corticomotoneuronal cells. Proc Natl Acad Sci 106:918-923.

Riemann BL, Lephart SM (2002) The Sensorimotor System, Part I: The Physiologic Basis of Functional Joint Stability. J Athl Train 37:71.

Roll J, Vedel J (1982) Kinaesthetic role of muscle afferents in man, studied by tendon vibration and microneurography. Exp brain Res 47:177-190.

Roux F-E, Djidjeli I, Durand J-B (2018) Functional architecture of the somatosensory homunculus detected by electrostimulation. J Physiol 596:941-956.

Schibli L, Gandia R, Buck R, Staempfli P, Meier M, Schuetz P (2021) MR-safe multisegmental vibration device for cortical mapping of paraspinal afferent input. TechRXiv https://doi.org/10.36227/techrxiv.17161883.

Schmid S, Bangerter C, Schweinhardt P, Meier ML (2021) Identifying Motor Control Strategies and Their Role in Low Back Pain: A Cross-Disciplinary Approach Bridging Neurosciences With Movement Biomechanics. Front Pain Res 2:715219. 
Seizova-Cajic T, Smith JL, Taylor JL, Gandevia SC (2007) Proprioceptive movement illusions due to prolonged stimulation: Reversals and aftereffects. PLoS One 2:e1037.

Smith SM, Jenkinson M, Woolrich MW, Beckmann CF, Behrens TEJ, Johansen-Berg H, Bannister PR, De Luca M, Drobnjak I, Flitney DE, Niazy RK, Saunders J, Vickers J, Zhang Y, De Stefano N, Brady JM, Matthews PM (2004) Advances in functional and structural MR image analysis and implementation as FSL. Neuroimage 23 Suppl 1:S208-19.

Stephan KE, Petzschner FH, Kasper L, Bayer J, Wellstein K V., Stefanics G, Pruessmann KP, Heinzle J (2019) Laminar fMRI and computational theories of brain function. Neuroimage 197:699-706.

Thiese MS, Hegmann KT, Wood EM, Garg A, Moore JS, Kapellusch J, Foster J, Ott U (2014) Prevalence of low back pain by anatomic location and intensity in an occupational population. BMC Musculoskelet Disord 15:1-11.

Tong MH, Mousavi SJ, Kiers H, Ferreira P, Refshauge K, Dieën J van (2017) Is There a Relationship Between Lumbar Proprioception and Low Back Pain? A Systematic Review With Meta-Analysis. Arch Phys Med Rehabil 98:120-136.e2.

Tsao H, Danneels L, Hodges PW (2011a) Individual fascicles of the paraspinal muscles are activated by discrete cortical networks in humans. Clin Neurophysiol 122:1580-1587.

Tsao H, Danneels LA, Hodges PW (2011b) ISSLS prize winner: Smudging the motor brain in young adults with recurrent low back pain. Spine (Phila Pa 1976) 36:1721-1727.

Tustison NJ, Avants BB, Cook PA, Zheng Y, Egan A, Yushkevich PA, Gee JC (2010) N4ITK: Improved N3 bias correction. IEEE Trans Med Imaging 29:1310-1320.

Van Dieën JH, Flor H, Hodges PW (2017) Low-Back Pain Patients Learn to Adapt Motor Behavior With Adverse Secondary Consequences. Exerc Sport Sci Rev 45:223-229.

Van Dieën JH, Peter Reeves N, Kawchuk G, Van Dillen LR, Hodges PW (2019) Motor Control Changes in Low Back Pain: Divergence in Presentations and Mechanisms 49:370-379.

Vedel JP, Roll JP (1982) Response to pressure and vibration of slowly adapting cutaneous mechanoreceptors in the human foot. Neurosci Lett 34:289-294.

Vos $\mathrm{T}$ et al. (2017) Global, regional, and national incidence, prevalence, and years lived with disability for 328 diseases and injuries for 195 countries, 1990-2016: a systematic analysis for the Global Burden of Disease Study 2016. Lancet 390:1211-1259.

Wand BM, Parkitny L, O’Connell NE, Luomajoki H, McAuley JH, Thacker M, Moseley GL (2011) Cortical changes in chronic low back pain: Current state of the art and implications for clinical practice. Man Ther 16:15-20.

Weerakkody NS, Mahns DA, Taylor JL, Gandevia SC (2007) Impairment of human proprioception by high-frequency cutaneous vibration. J Physiol 581:971-980.

Winter DA (1995) Human balance and posture control during standing and walking. Gait Posture 3:193-214.

Zhang Y, Brady M, Smith S (2001) Segmentation of brain MR images through a hidden Markov random field model and the expectation-maximization algorithm. IEEE Trans Med Imaging 20:45-57. 